\title{
Diferencias de género en el conocimiento y las percepciones del cambio climático entre adolescentes. Metaanálisis
}

\author{
Gender Differences in Adolescent's Climate Change \\ Knowledge and Perceptions. Meta-Analysis
}

\section{Antonio García-Vinuesa, María Lucía Iglesias da Cunha, Rita Gradaílle Pernas}

Grupo de Investigación en Pedagogía Social y Educación Ambiental, SEPA-interea (Gl-1447) Universidade de Santiago de Compostela

\section{Resumen}

\begin{abstract}
¿Existen diferencias de conocimiento acerca del cambio climático entre adolescentes mujeres y hombres? ¿Cómo perciben los adolescentes la crisis climática en función del género? Con el fin de dar respuesta a estas preguntas se realizó un metaanálisis con una muestra de 84 artículos internacionales seleccionados con la metodología de revisión bibliográfica sistematizada, para lo cual se identificaron aquellos en los que — de acuerdo con la variable de géneroexistían diferencias de conocimiento, percepciones y/o valoraciones entre los adolescentes que cursaban educación secundaria. Del total de artículos — que se concentró entre 1993 y 2017- 26 de ellos (30,95\%) abordaban las diferencias de género en relación con el cambio climático. El metaanálisis reveló que las mujeres obtenían menores puntuaciones que los hombres respecto de los conocimientos en esta temática, con una diferencia aumentada conforme se avanzaba en los respectivos cursos de educación secundaria, aunque atribuían un mayor riesgo a la crisis climática, presentaban mayor preocupación por el problema y, a diferencia de los hombres que evidenciaban una cosmovisión más tecnicista, estas mostraron mayor disposición ecocéntrica para aceptar las medidas contra la crisis climática.
\end{abstract}

Palabras clave: adolescentes, cambio climático, estudiantes, género, metaanálisis.

\footnotetext{
Correspondencia a:

Antonio García-Vinuesa

Facultad de Ciencias de Educación - Campus Vida (15782), Universidade de Santiago de Compostela, Despacho 33, Módulo A, A Coruña, España.

a.garcia.vinuesa@usc.es

Financiamiento asociado: Educación para el cambio climático en educación secundaria: investigación aplicada sobre representaciones y estrategias pedagógicas en la transición ecológica. Código de referencia: RTI2018-094074-B-I00 (RESCLIMA-EDU2). Convocatoria de 2018 de Proyectos de I+D+i Retos Investigación del Programa Estatal de I+D+I orientada a los retos de la sociedad. Ministerio de Ciencia, Innovación y Universidades, Gobierno de España.
}

(C) $2020 \mathrm{PEL}$, http://www.pensamientoeducativo.org - http://www.pel.cl 


\section{Abstract}

Are there differences between adolescent men and women in terms of knowledge about climate change? How do adolescents perceive the climate crisis depending on their gender? In order to answer these questions, we carried out a meta-analysis on a sample of 84 international papers selected by conducting a systematic review methodology. To achieve that aim, we identified studies that showed differences according to the gender variable in terms of knowledge, perceptions, and/or opinions among adolescents pursuing secondary education. A total of 26 out of 84 papers (30.95\%) — concentrated between 1993 and 2017—addressed gender differences regarding climate change. The meta-analysis revealed that women score lower than men on knowledge about climate change, with the difference increasing as they move forward in their respective secondary education courses. However, women are found to attribute greater risk to the climate crisis, they are more concerned about the problem, and—unlike men, who have a more technical worldview—-they show greater ecocentric willingness to accept measures to address it.

Keywords: adolescents, climate change, gender, meta-analysis, students.

\section{Introducción}

Debido a su complejidad y multidimensionalidad el cambio climático (en adelante CC) ha sido incluido como contenido curricular en un espacio entre la enseñanza de las ciencias y la educación ambiental. En el primer caso, este se fundamenta en el conocimiento de sus bases físico-químicas, abordándolo como un fenómeno principalmente atmosférico (Serantes-Pazos, 2017) a partir de enfoques alfabetizadores basados en la teoría del cambio conceptual (Oliva, 1999). En la segunda instancia, este se basaría en el constructivismo social, que busca comprender la construcción de significados subjetivos de las relaciones entre las sociedades humanas y el medio ambiente, a través de procesos sociales, históricos y normas culturales (Creswell, 2013).

La larga trayectoria de ambas disciplinas se evidencia en la prolífica literatura, en la que podemos encontrar estudios, revisiones y metaanálisis relativos a las diferencias de género existentes en los conocimientos, percepciones o disposiciones hacia los contenidos científico-técnicos y socioambientales, lo que permite realizar un acercamiento a nuestro tópico de estudio: el cambio climático y las diferencias de género en su representación. Al respecto, Fisher, Thompson y Brookes (2020) realizaron una revisión de la literatura científica con el objetivo de comprender qué factores influían en el bajo número (inferior al 15\%) de mujeres australianas matriculadas en cursos universitarios relacionados con disciplinas de la ciencia, tecnología, ingenierías y matemáticas (STEM, por sus siglas en inglés). En su investigación, de un total de 26 estudios, se identificaron 20 de ellos con un porcentaje mayor de mujeres que declaraba un nivel de autoeficacia menor que los hombres en cuanto a habilidades y capacidades científicas; incluso en áreas donde el porcentaje de mujeres matriculadas es mayor, por ejemplo en Biología.

Así, y pese a que el estudio no indagaba explícitamente en rasgos de autoeficacia, los resultados mostraron menor confianza de las mujeres en sus conocimientos y habilidades. Las autoras destacan que la percepción de autoeficacia es uno de los principales elementos de motivación, por encima de la posesión de conocimientos, lo cual constituye un factor determinante de desigualdad de género en este tipo de experiencias educativas. En este sentido, Schönfelder y Bogner (2020) analizaron las interacciones existentes entre la educación científica y los 
valores ambientales, tópico transversal que suele relacionarse con la enseñanza de las ciencias. En este estudio se evidencia un mayor nivel de autoeficacia ante la resolución de problemas relacionados con contenidos científicotécnicos en los hombres, lo que podría influir en una motivación intrínseca menor de las mujeres hacia las ciencias.

Por su parte, McCright (2010) realizó un análisis entre 2001 y 2008 a partir de diversas demoscopias (gallup polls) centradas en aspectos relacionados con el medio ambiente. Los datos revelaron diferencias en el conocimiento científico o ecológico según el género, siendo ellas las que declaraban niveles inferiores de conocimientos, diferencia que se incrementaba con el paso de los años y a medida que ascendían de nivel educativo. Según Finucane, Slovic, Mertz, Flynn y Satterfield (2000), esta circunstancia se relaciona con una menor confianza en sus habilidades y conocimientos científicos, lo que condiciona las futuras elecciones en sus orientaciones académicas y aumentando — más, si cabe— la brecha de género.

Por otro lado, diferentes autores defienden que una menor disposición hacia las ciencias y las tecnologías se relaciona con determinados métodos de enseñanza en los que las prácticas individualistas y competitivas - con un interés menor para las mujeres_, copan gran parte de los procesos de enseñanza-aprendizaje de las ciencias. De igual modo, existen explicaciones que subrayan la falta de figuras científicas femeninas relevantes, así como las expectativas de compatibilizar una carrera científica profesional con la conformación de una familia (Dijkstra \& Goedhart, 2011).

En relación con este punto, Fisher et al. (2020) identificaron que 15 de los 36 estudios seleccionados exploraban las valoraciones de los procesos de enseñanza-aprendizaje en disciplinas STEM, en los que se destaca la ansiedad que produce la evaluación de este tipo de conocimientos mayormente en las mujeres y que ello presenta una relación directa con los bajos niveles de autoeficacia mencionados con anterioridad. Por otro lado, este estudio mantiene la tendencia de aquellos que identifican rasgos de masculinización en las disciplinas STEM, como el estereotipo de "geek" de la tecnología con el que las estudiantes declararon no sentirse identificadas. Una conclusión que ha sido reforzada y detallada por Cheryan, Plaut, Davies y Steele (2009) en relación con los motivos por los que las mujeres no se sienten atraídas por las ciencias de la computación. Estos autores acuñaron el concepto de "ambiente de pertenencia" (ambient belonging) y desarrollaron una simulación que permitió identificar un bajo sentido de pertenencia de las mujeres a ese tipo de lugares, claramente estereotipados por personas interesadas por la informática.

McCright (2010) también sugiere que ellas declaran una mayor preocupación por los problemas ambientales locales que afectan a la salud y que comportan posibles riesgos para la seguridad de su comunidad, percepciones que disminuyen frente a problemas alejados de las fronteras locales, y que se refuerzan ante situaciones que pueden conllevar dificultades. Por su parte, Jenkins y Pell (2006) constataron que las mujeres mostraban mayor esperanza en que sus acciones individuales sirvieran para mejorar los problemas ambientales más graves, aunque mostraron menor confianza en la ciencia y la tecnología como solución a la crisis ambiental.

Igualmente encontramos revisiones y metaanálisis donde se exploraron diversos tópicos relacionados con la enseńanza del CC; sin embargo, solo en dos de estas investigaciones se evidenciaron diferencias relativas al género. Uno de estos casos fue el estudio de Bozdoğan (2011) que indicó que las mujeres tenían actitudes más positivas y eran más sensibles a los problemas ambientales, sin que se constatara una correlación entre los estilos de enseñanza del profesorado. Para Monroe, Plate, Oxarart, Bowers y Chaves (2019), en tanto, las mujeres - a diferencia de los hombres, que no presentan sesgos geográficos - aprenden más a través de ejemplos locales en lugar de ejemplos globales.

1. El término "geek" hace referencia a una persona un tanto fanática de la tecnología, los computadores o la informática. Su uso es próximo al ya más reconocible de friki o nerd, pero en cada contexto cultural puede tener variaciones en las características específicas de las personas que reciben este apelativo. 
Planteado este escenario, la literatura sugiere algunas diferencias de género respecto de la adquisición o disposición de contenidos científicos al evidenciar que las mujeres presentan una cosmovisión más ecocéntrica frente a la tecnocéntrica de los varones (Jenkins \& Pell, 2006; Schönfelder \& Bogner, 2020).

\section{Objetivo del estudio}

El estudio exploró los resultados ofrecidos por la literatura científica en cuanto a las diferencias de género existentes en estudiantes de educación secundaria (12-18 años) respecto del conocimiento y representación del CC, como síntesis del saber generado en torno a este tópico en el período comprendido entre 1993 y 2017.

\section{Metodología}

La revisión bibliográfica sistematizada (RBS) es una estrategia de búsqueda normalizada que permite realizar una investigación bibliográfica relativa a un tópico concreto. La secuenciación y sistematización de la estrategia de exploración (selección de fuentes y tesauros, fechas de acceso, algoritmos de búsqueda, criterios de inclusión/exclusión, procesos de depuración, etc.) posibilita que la información sea revisada, replicada y actualizada. La singularidad de esta metodología, cuyo impulso se sitúa a finales de 1970, permite identificar investigaciones constituyentes de un campo de estudio concreto, además de sintetizar sus resultados (Littell, Corcoran \& Pillai, 2008).

Su aplicación al campo de las ciencias sociales deviene de su fácil e instantáneo acceso a una gran cantidad de información digitalizada proporcionada por las actuales bases de datos, así como algunas guías para su realización, como la declaración PRISMA (Preferred Reporting Items for Systematic reviews and Meta-Analyses)². Si bien se trata de una metodología ideada para las ciencias biomédicas, ofrece un protocolo estandarizado para mejorar la calidad de los informes de metaanálisis, además de adaptarse a otras disciplinas científicas. Sus características de transparencia, replicabilidad y actualización permiten que sus hallazgos — que representan las evidencias de otros estudios - puedan ser supervisados y evaluados por el resto de la comunidad científica interesada.

Con todo, una RBS puede comportar — o no- la realización de un metaanálisis. La diferencia radica en que la revisión tiene como objetivo identificar y sintetizar estudios de una cuestión en particular; mientras que el metaanálisis, en su expresión más cuantitativa, es un conjunto de técnicas estadísticas que permiten combinar los resultados de diversos estudios y obtener medidas globales. En este sentido, aunque diferentes, es recomendable que un metaanálisis se realice posteriormente a la RBS, con el fin de evitar sesgos (Littell et al., 2008). En este caso, se ha realizado un metaanálisis cualitativo (Timulak, 2009) por medio de la utilización de técnicas de análisis de otro tipo de revisiones, como las revisiones narrativas o las síntesis conceptuales, con la intención de profundizar en los resultados e ir más allá de una explicación meramente causal (Petticrew \& Roberts, 2008).

\section{Revisión bibliográfica sistematizada}

El diagrama de flujo de la Figura 1 muestra una síntesis de los pasos de la RBS, que identifica las investigaciones de relevancia en torno al estudio del CC, con estudiantes de educación secundaria (12-18 años) como participantes y con un compendio de artículos de entre 1993 y 2017. Una amplia y detallada explicación del proceso llevado a cabo puede encontrarse en García-Vinuesa y Meira-Cartea (2019), donde se ofrecen los resultados bibliométricos y el diseño metodológico detallado de esta RBS.

2. Elementos Preferentes de Informes para Revisiones Sistematizadas y Metaanálisis. 


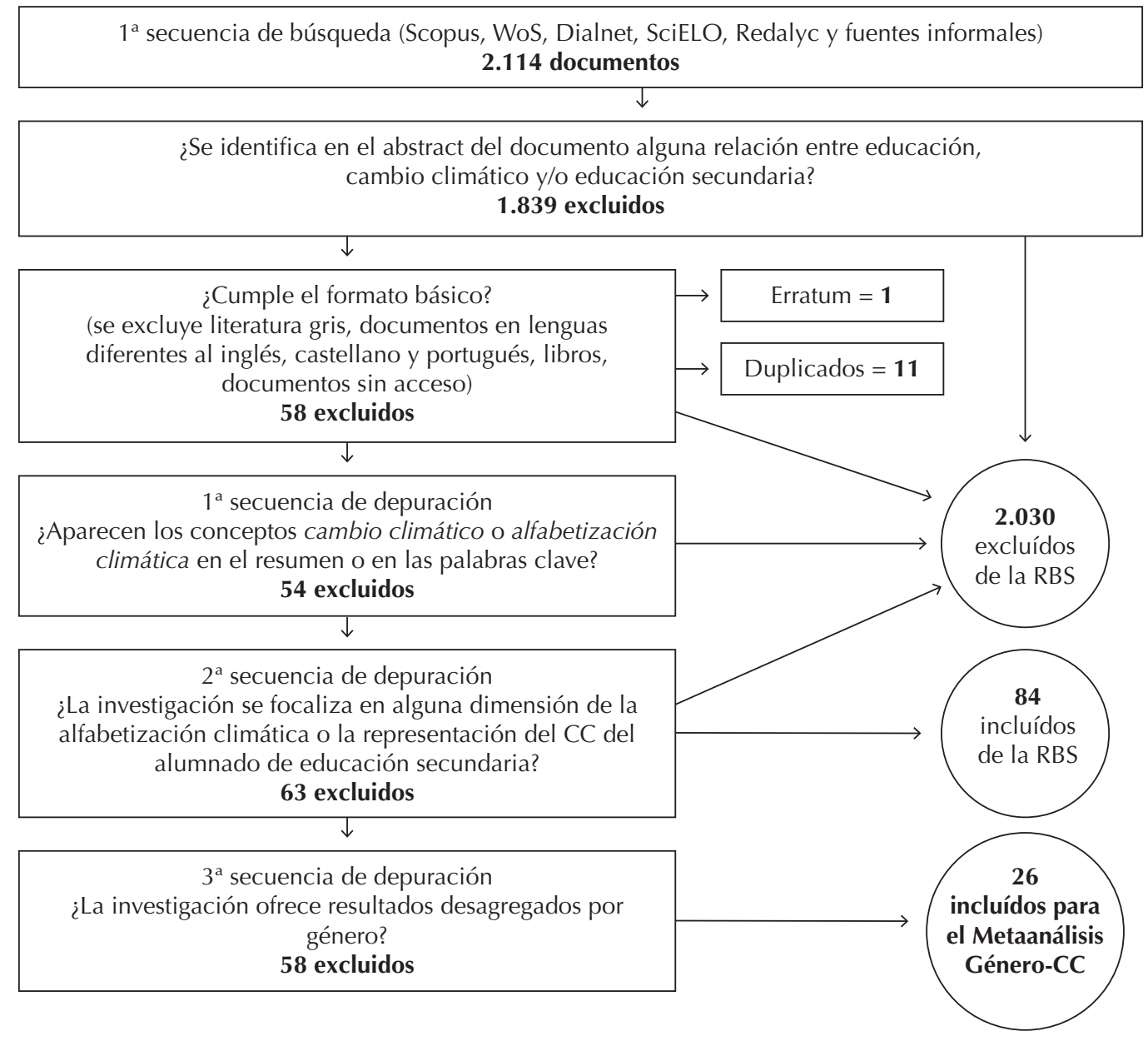

Figura 1. Diagrama de flujo de la RBS (García-Vinuesa y Meira-Cartea, 2019).

Fuente: Elaboración propia

\section{Metaanálisis cualitativo}

Una misma RBS puede contener múltiples metaanálisis (Littell et al., 2008). En este caso concreto, la realización de este metaanálisis cualitativo comportó una tercera secuencia de depuración de los resultados de la RBS (Figura 1). Así, se realizó una exploración automática de términos clave en los 84 artículos, al tiempo que se analizó el escenario textual en el que aparecían, con el fin de identificar los estudios que ofrecían resultados desde una perspectiva de género. De este modo, se utilizaron los siguientes términos de búsqueda: female, women, woman, girl y gender (en inglés); femenino, mujer, nińa y género (en castellano); garota, rapariga, mulher, menina y género (en portugués); y muller, nena y xénero (en gallego). Con el fin de comprobar que ningún estudio quedara excluido, se leyeron todos los apartados relativos a los resultados y a la discusión de las investigaciones incluidas en la RBS, proceso que confirmó los resultados iniciales y permitió la identificación de un total de 26 estudios. 


\section{Análisis de los resultados}

En la Tabla 1 se presenta la selección de los 26 estudios que muestran diferencias de género en relación con la comprensión del CC, y ofrece una síntesis general de los resultados para caracterizar los estudios en función del país de origen de los participantes, niveles educativos, objeto de estudio y diferencias estadísticamente significativas existentes entre ambos géneros.

De igual modo, se establecieron categorías en función de la descripción del objeto de estudio y de los diferentes elementos explorados en cada investigación, lo cual representó una difícil tarea de estructuración debido a la diversidad terminológica utilizada para denominar los elementos de representación del CC en los distintos estudios, ya sea desde el punto de vista de las percepciones, las concepciones, los conocimientos o las creencias, tal y como ya advertía Cubero (1994). Esta categorización consideró las justificaciones de los investigadores, así como la metodología empleada, de tal modo que durante el proceso de registro de los datos surgieron tres categorías analíticas: una que engloba los resultados de estudios que exploraron diversos conocimientos específicos; la segunda recoge la información referente a percepciones y valoraciones personales; y la tercera reúne elementos relacionados con los procesos de enseñanza-aprendizaje. Estos resultados agrupados en categorías presentan aquellas variables o tópicos donde se obtuvieron diferencias estadísticamente significativas para $p<0,05$ o $p<0,01$.

\section{Resultados}

De los 26 estudios identificados, tres de ellos se basan en un diseño metodológico cualitativo frente a 23 investigaciones con diseños cuantitativos (Tabla 1). Con la excepción de dos casos en los que las muestras fueron pequeñas (24 y 29 participantes) — coincidentes con los estudios de corte cualitativo (Lin, 2017; Rye, Rubba \& Wiesenmayer, 1997)—, las investigaciones consideraron muestras de gran tamaño, con un rango mínimo de 188 y un máximo de 1.532 participantes. En tres estudios (Ambusaidi, Boyes, Stanisstreet \& Taylor, 2012; Boyes, Chuckran y Stanisstreet, 1993; Boyes \& Stanisstreet, 2012) participaron estudiantes de primaria (1012 años). En cuanto al objeto de estudio encontramos diversos elementos de la representación del CC, lo que permitió establecer tres grandes categorías:

a) Conocimientos del CC. Una primera categoría concerniente a la información en torno al CC que poseen en forma de conocimiento objetivado. Estos estudios indagan en aquellos conocimientos enmarcados en dos de las tipologías clásicas: conceptuales y actitudinales (Tablas 2 y 3 ).

b) Percepciones y valoraciones personales. Esta categoría hace referencia a procesos subjetivos de la esfera de las percepciones y valoraciones personales (Tabla 4). En este caso los autores de los artículos de la muestra resultante ofrecen una amplia variedad de términos para referirse a sus objetos de estudio, tales como concepción, percepción, comprensión, ideas, cosmovisiones, creencias, actitudes y preocupaciones, entre otros.

c) Procesos de enseñanza-aprendizaje. Esta categoría engloba resultados relacionados con los procesos de enseñanza-aprendizaje. 
Tabla 1

Selección de investigaciones donde el objeto de estudio indaga en elementos de la representación del CC en estudiantes de 12 a 18 años

\begin{tabular}{|c|c|c|c|c|c|c|c|c|c|c|c|}
\hline Estudio & País & $\mathrm{N}$ & G7 & G8 & G9 & G10 & G11 & G12 & Objeto de estudio & Diferencias & Metodología \\
\hline $\begin{array}{c}\text { Boyes et al. } \\
(1993)^{*}\end{array}$ & Inglaterra & 702 & * & * & * & * & & & $\begin{array}{l}\text { Comprensión del } \\
\text { efecto invernadero }\end{array}$ & No & Cuantitativa \\
\hline Rye et al. (1997) & EE.UU. & 24 & * & $*$ & $*$ & & & & $\begin{array}{l}\text { Comprensión del } \\
\text { calentamiento } \\
\text { global (CG) }\end{array}$ & No & Cualitativa \\
\hline $\begin{array}{c}\text { Daniel, } \\
\text { Stanisstreet y } \\
\text { Boyes (2004) }\end{array}$ & Inglaterra & 582 & & $*$ & & $*$ & & & $\begin{array}{l}\text { Ideas sobre } \\
\text { mitigación del CG }\end{array}$ & Sí & Cuantitativa \\
\hline $\begin{array}{c}\text { Boyes, } \\
\text { Stanisstreet y } \\
\text { Yongling }(2008)\end{array}$ & China & 676 & & * & & & $*$ & & $\begin{array}{l}\text { Creencias: } \\
\text { acciones de } \\
\text { mitigación }\end{array}$ & Sí & Cuantitativa \\
\hline $\begin{array}{c}\text { Boyes, Skamp } \\
\text { y Stanisstreet } \\
\quad(2009)\end{array}$ & Australia & 500 & * & $*$ & * & $*$ & & & $\begin{array}{l}\text { Creencias: } \\
\text { acciones de } \\
\text { mitigación }\end{array}$ & Sí & Cuantitativa \\
\hline $\begin{array}{c}\text { Rodríguez, Boyes } \\
\text { y Stanisstreet } \\
(2010)\end{array}$ & España & 1.460 & * & * & $*$ & $*$ & & & $\begin{array}{l}\text { Creencias y } \\
\text { actitudes para la } \\
\text { acción }\end{array}$ & Sí & Cuantitativa \\
\hline $\begin{array}{l}\text { Chhokar, Dua, } \\
\text { Taylor, Boyes } \\
\text { y Stanisstreet } \\
\text { (2011) }\end{array}$ & India & 768 & * & $*$ & $*$ & * & & & $\begin{array}{l}\text { Creencias y } \\
\text { actitudes para la } \\
\text { acción }\end{array}$ & No & Cuantitativa \\
\hline $\begin{array}{c}\text { Dijkstra y } \\
\text { Goedhart (2011) }\end{array}$ & Europa & 1.370 & * & $*$ & * & * & * & * & $\begin{array}{l}\text { Disposición hacia } \\
\text { la ciencia }\end{array}$ & Sí & Cuantitativa \\
\hline $\begin{array}{c}\text { Kılınç, Boyes } \\
\text { y Stanisstreet } \\
(2011)\end{array}$ & Turquía & 897 & * & * & * & * & & & $\begin{array}{l}\text { Creencias y } \\
\text { actitudes para la } \\
\text { acción }\end{array}$ & Sí & Cuantitativa \\
\hline $\begin{array}{l}\text { Malandrakis, } \\
\text { Boyes y } \\
\text { Stanisstreet } \\
\text { (2011) }\end{array}$ & Grecia & 1.444 & * & * & * & * & & & $\begin{array}{l}\text { Creencias y } \\
\text { actitudes para la } \\
\text { acción }\end{array}$ & Sí & Cuantitativa \\
\hline $\begin{array}{c}\text { Liarakou, } \\
\text { Athanasiadis y } \\
\text { Gavrilakis (2011) }\end{array}$ & Grecia & 626 & & * & * & * & * & & Conocimientos & Sí & Cuantitativa \\
\hline $\begin{array}{l}\text { Ambusaidi et al. } \\
(2012)^{*}\end{array}$ & Omán & 1.532 & * & $*$ & * & $*$ & * & $*$ & $\begin{array}{l}\text { Creencias y } \\
\text { actitudes para la } \\
\text { acción }\end{array}$ & Sí & Cuantitativa \\
\hline $\begin{array}{c}\text { Boyes y } \\
\text { Stanisstreet } \\
(2012)^{*}\end{array}$ & Inglaterra & 961 & * & * & * & * & & & $\begin{array}{l}\text { Creencias y } \\
\text { actitudes para la } \\
\text { acción }\end{array}$ & Sí & Cuantitativa \\
\hline $\begin{array}{c}\text { Dijkstra y } \\
\text { Goedhart (2012) }\end{array}$ & Europa & 671 & * & * & $*$ & * & * & * & $\begin{array}{l}\text { Conductas pro- } \\
\text { ambientales, } \\
\text { conocimientos y } \\
\text { actitudes }\end{array}$ & Sí & Cuantitativa \\
\hline
\end{tabular}




\begin{tabular}{|c|c|c|c|c|c|c|c|c|c|c|c|}
\hline $\begin{array}{l}\text { Barros y Pinheiro } \\
\text { (2013) }\end{array}$ & Brasil & 323 & $*$ & * & * & * & * & * & $\begin{array}{l}\text { Comprensión del } \\
\text { CC }\end{array}$ & - & Cualitativa \\
\hline $\begin{array}{c}\text { Harker-Schuch y } \\
\text { Bugge-Henriksen } \\
\text { (2013) }\end{array}$ & $\begin{array}{c}\text { Austria/ } \\
\text { Dinamarca }\end{array}$ & $\begin{array}{c}188 \\
(361)\end{array}$ & & & & & * & & $\begin{array}{l}\text { Conocimientos y } \\
\text { opiniones }\end{array}$ & Sí & Cuantitativa \\
\hline $\begin{array}{l}\text { Yazdanparast et } \\
\text { al. (2013) }\end{array}$ & Irán & 1.035 & & & * & * & * & & $\begin{array}{l}\text { Conocimientos y } \\
\text { visiones del CC }\end{array}$ & Sí & Cuantitativa \\
\hline $\begin{array}{c}\text { Özdem, Dal, } \\
\text { Öztürk, Sönmez } \\
\text { \& Alper (2014) }\end{array}$ & Turquía & 646 & * & & & & & & $\begin{array}{l}\text { Preocupaciones, } \\
\text { experiencias, } \\
\text { creencias, } \\
\text { actitudes, valores, } \\
\text { etc. }\end{array}$ & Sí & Cuantitativa \\
\hline $\begin{array}{c}\text { Stevenson, } \\
\text { Peterson, Bondell, } \\
\text { Moore \& Carrier } \\
\text { (2014) }\end{array}$ & EE.UU. & 378 & $*$ & * & & & & & $\begin{array}{l}\text { Conocimientos y } \\
\text { riesgo }\end{array}$ & Sí & Cuantitativa \\
\hline $\begin{array}{l}\text { Bodzin y Fu } \\
\quad(2014)\end{array}$ & EE.UU. & 956 & & * & * & & & & $\begin{array}{l}\text { Conocimientos } \\
\text { sobre CC }\end{array}$ & Sí & Cuantitativa \\
\hline $\begin{array}{c}\text { Stevenson y } \\
\text { Peterson (2016) }\end{array}$ & EE.UU. & 1.267 & * & * & & & & & $\begin{array}{l}\text { Esperanza y } \\
\text { preocupación }\end{array}$ & Sí & Cuantitativa \\
\hline $\begin{array}{c}\text { Stevenson, } \\
\text { Peterson y } \\
\text { Bradshaw (2016) }\end{array}$ & EE.UU. & 369 & * & * & & & & & $\begin{array}{l}\text { Conocimientos y } \\
\text { creencias }\end{array}$ & Sí & Cuantitativa \\
\hline $\begin{array}{c}\text { Stevenson, } \\
\text { Peterson y } \\
\text { Bondell (2016) }\end{array}$ & EE.UU. & 426 & $*$ & * & & & & & $\begin{array}{l}\text { Creencias y } \\
\text { percepciones }\end{array}$ & Sí & Cuantitativa \\
\hline $\begin{array}{c}\text { Hermans y } \\
\text { Korhonen (2017) }\end{array}$ & Finlandia & 549 & & & * & & & & $\begin{array}{l}\text { Actitudes, visiones } \\
\text { y disposición }\end{array}$ & Sí & Cuantitativa \\
\hline Lin (2017) & China & 39 & & * & & & & & $\begin{array}{l}\text { Conocimientos y } \\
\text { acciones }\end{array}$ & Sí & Cualitativa \\
\hline $\begin{array}{l}\text { Stevenson, King, } \\
\text { Selm, Peterson y } \\
\text { Monroe (2017) }\end{array}$ & EE.UU. & 950 & * & * & & & & & $\begin{array}{l}\text { Creencias y } \\
\text { percepciones }\end{array}$ & Sí & Cuantitativa \\
\hline
\end{tabular}

Nota: G7 = 12-13 años; G8 = 13-14 años; G9 = 14-15 ańos; G10 = 15-16 años; G11 = 16-17 años; $\mathrm{G12}=17-18$ años.

* Participantes de estudios primarios.

Fuente: Elaboración propia.

A la vista de los resultados presentados, el $88,46 \%$ de los estudios ofrecen diferencias significativas entre mujeres y hombres adolescentes (Tabla 1). En el caso del estudio de Barros y Pinheiro (2013), este se codificó considerando que no existían diferencias significativas, ya que las autoras indican que sus resultados no permiten hacer la distinción por géneros; no obstante, exponen una diferencia en relación con los mensajes de conservación que emitieron los participantes, siendo estos mayoritariamente emitidos por mujeres.

De los datos presentados en las Tablas 2, 3 y 4, destaca que en los ítems o en los contenidos específicos en los que los estudios reportan diferencias estadísticamente significativas, las mujeres obtienen mayores porcentajes (ocho de cada diez casos). Sin embargo, este dato debe ser matizado respecto de la subcategoría de conocimientos 
conceptuales (Tabla 2), ya que aunque los hombres —en el 25\% de los casos- obtuvieron mayores porcentajes de respuesta correcta, las mujeres obtuvieron el mismo porcentaje de respuesta incorrecta, siendo en esta sección donde las diferencias significativas se distribuyen de forma igualitaria entre ambos géneros. Cabe añadir que en los dos estudios donde se ofrecen los valores de significación al comparar los resultados totales de un cuestionario sobre conocimientos generales del CC en función del género (Harker-Schuch \& Bugge-Henriksen, 2013; Yazdanparast et al., 2013), se confirmó que los hombres obtuvieron mejores puntuaciones que las mujeres.

\section{Conocimientos acerca del CC}

En esta categoría se han agrupado los estudios que, de forma general o parcial, exploran diversos conocimientos relacionados con el CC. En la Tabla 2 únicamente se registran los resultados con significación estadística para $p<0,05$ o $p<0,01$, y en los que únicamente se consideró la variable género como factor.

Esta categoría, tal y como se ha indicado con anterioridad, se ha dividido en dos subcategorías: conocimientos conceptuales y actitudinales.

Conocimientos conceptuales. La Tabla 2 ofrece los contenidos conceptuales específicos en torno al CC que obtuvieron diferencias estadísticamente significativas al comparar los resultados en función del género.

Tabla 2

Contenidos relacionados con conocimientos conceptuales de CC con significación estadistica al comparar resultados según género

\begin{tabular}{|c|c|c|c|c|}
\hline Estudio & $\begin{array}{l}\text { Objeto de } \\
\text { estudio }\end{array}$ & Contenido específico & M & $\mathrm{H}$ \\
\hline \multirow{7}{*}{$\begin{array}{l}\text { Daniel et al. } \\
\text { (2004) }\end{array}$} & \multirow{7}{*}{$\begin{array}{l}\text { Creencias sobre } \\
\text { acciones para la } \\
\text { mitigación del } \\
\text { CC }\end{array}$} & Reducir la contaminación marina* & $53 \%$ & $41 \%$ \\
\hline & & Reducir la basura de los ríos* & $53 \%$ & $45 \%$ \\
\hline & & Reducir la producción nuclear* & $74 \%$ & $63 \%$ \\
\hline & & $\begin{array}{l}\text { Reducir las emisiones de CFCs* } \\
\text { (clorofluorocarbonos) }\end{array}$ & $72 \%$ & $63 \%$ \\
\hline & & Reducir el uso de petróleo & $62 \%$ & $68 \%$ \\
\hline & & Reducir el uso de carbón & $69 \%$ & $71 \%$ \\
\hline & & Usar vehículos eléctricos & $75 \%$ & $82 \%$ \\
\hline \multirow{5}{*}{$\begin{array}{l}\text { Boyes et al. } \\
\text { (2008) }\end{array}$} & \multirow{5}{*}{$\begin{array}{l}\text { Creencias sobre } \\
\text { acciones para la } \\
\text { mitigación }\end{array}$} & Reducir la contaminación marina* & $73 \%$ & $64 \%$ \\
\hline & & Reducir la basura* & $61 \%$ & $50 \%$ \\
\hline & & Proteger especies exóticas* & $56 \%$ & $46 \%$ \\
\hline & & Importancia de la educación & $83 \%$ & $76 \%$ \\
\hline & & Uso de gasolina sin plomo* & $79 \%$ & $71 \%$ \\
\hline \multirow{3}{*}{$\begin{array}{l}\text { Boyes et al. } \\
\text { (2009) }\end{array}$} & \multirow{3}{*}{$\begin{array}{l}\text { Acciones para la } \\
\text { mitigación }\end{array}$} & Usar automóviles más pequeños & $59 \%$ & $46 \%$ \\
\hline & & Apagar los dispositivos electrónicos & $53 \%$ & $36 \%$ \\
\hline & & Incrementar el uso de energía nuclear & $40 \%$ & $30 \%$ \\
\hline $\begin{array}{l}\text { Rodríguez et } \\
\text { al. (2010) }\end{array}$ & $\begin{array}{l}\text { Creencias sobre } \\
\text { acciones }\end{array}$ & Efectividad de las plantaciones de árboles & & $*$ \\
\hline
\end{tabular}




\begin{tabular}{|c|c|c|c|c|}
\hline \multirow{8}{*}{$\begin{array}{l}\text { Kilınç et al. } \\
\quad(2011)\end{array}$} & \multirow{8}{*}{$\begin{array}{c}\text { Ideas/creencias } \\
\text { sobre acciones } \\
\text { para la } \\
\text { mitigación }\end{array}$} & Producción de energía nuclear & $57 \%$ & $49 \%$ \\
\hline & & Mejorar la aislación del hogar & $63 \%$ & $52 \%$ \\
\hline & & Reducir el consumo energético doméstico & $75 \%$ & $60 \%$ \\
\hline & & Reducir el uso de fertilizantes artificiales & $71 \%$ & $56 \%$ \\
\hline & & Usar electrodomésticos más eficientes & $63 \%$ & $56 \%$ \\
\hline & & Importancia de la educación & $85 \%$ & $79 \%$ \\
\hline & & Comprar menos productos de moda nuevos & $27 \%$ & $34 \%$ \\
\hline & & Usar energías renovables & $60 \%$ & $66 \%$ \\
\hline \multirow{5}{*}{$\begin{array}{l}\text { Liarakou et al. } \\
\quad(2011)\end{array}$} & \multirow{5}{*}{ Conocimientos } & $\begin{array}{l}\text { La temperatura del planeta está } \\
\text { aumentando }\end{array}$ & $* *$ & \\
\hline & & La capa de hielo polar está aumentando* & ** & \\
\hline & & $\begin{array}{l}\text { CC intensificará los fenómenos } \\
\text { meteorológicos extremos }\end{array}$ & ** & \\
\hline & & El CC afectará la producción de alimentos & ** & \\
\hline & & $\begin{array}{l}\text { Las lámparas incandescentes ahorran } \\
\text { energía }\end{array}$ & & $* *$ \\
\hline \multirow{10}{*}{$\begin{array}{l}{ }^{* * *} \text { Ambusaidi } \\
\text { et al. (2012) }\end{array}$} & \multirow{10}{*}{$\begin{array}{c}\text { Ideas/creencias } \\
\text { sobre acciones } \\
\text { para la } \\
\text { mitigación }\end{array}$} & El CC es un fenómeno real & $82 \%$ & $78 \%$ \\
\hline & & Comer menos carne & $28 \%$ & $36 \%$ \\
\hline & & Aumentar la generación de energía nuclear & $53 \%$ & $62 \%$ \\
\hline & & Usar automóviles más eficientes & $70 \%$ & $62 \%$ \\
\hline & & Reducir el uso de fertilizantes artificiales & $60 \%$ & $54 \%$ \\
\hline & & Desconectar dispositivos que no se usen & $61 \%$ & $54 \%$ \\
\hline & & Reciclar* & $58 \%$ & $52 \%$ \\
\hline & & $\begin{array}{l}\text { Incrementar procesos de educación } \\
\text { ambiental }\end{array}$ & $77 \%$ & $72 \%$ \\
\hline & & Construir más acuerdos internacionales & $77 \%$ & $70 \%$ \\
\hline & & Aumentar la legislación ambiental & $77 \%$ & $70 \%$ \\
\hline $\begin{array}{c}\text { Harker-Schuch } \\
\text { y Bugge- } \\
\text { Henriksen } \\
(2013)\end{array}$ & $\begin{array}{l}\text { Conocimientos/ } \\
\text { opiniones }\end{array}$ & Aciertos totales en cuestionario & $50,1 \%$ & $55,6 \%$ \\
\hline $\begin{array}{l}\text { Yazdanparast } \\
\text { et al. (2013) }\end{array}$ & Conocimientos & $\begin{array}{l}\text { Puntuación total sobre conocimientos del } \\
\text { CC }\end{array}$ & & $* *$ \\
\hline
\end{tabular}

Nota: Se presentan los resultados que ofrecieron diferencias estadísticamente significativas para $\mathrm{p}<0,05$.

* El contenido expresa un conocimiento errado o concepción alternativa.

** El estudio no ofrece los porcentajes desagregados, pero indica que un mayor número de mujeres/hombres indicaron esta opción.

*** En Omán las aulas son diferenciadas por sexo.

Fuente: elaboración propia. 
Conocimientos actitudinales. En este subapartado se ofrecen los resultados de los estudios que exploraban la disposición a actuar y/o actitudes en relación con distintas dimensiones del CC.

Tabla 3

Contenidos relacionados con conocimientos actitudinales del CC con significación estadistica al comparar por género

\begin{tabular}{|c|c|c|c|c|}
\hline Estudio & Objeto de estudio & Contenido específico & M & $\mathrm{H}$ \\
\hline \multirow{8}{*}{$\begin{array}{l}\text { Boyes et al. } \\
\text { (2009) }\end{array}$} & \multirow{8}{*}{$\begin{array}{l}\text { Ideas/creencias sobre } \\
\text { acciones para la } \\
\text { mitigación del CC }\end{array}$} & Pagar más por una casa bien aislada & $37 \%$ & $31 \%$ \\
\hline & & Apagar los dispositivos electrónicos & $79 \%$ & $66 \%$ \\
\hline & & Modificar la dieta (menos consumo de carne) & $28 \%$ & $12 \%$ \\
\hline & & Reciclar* & $70 \%$ & $58 \%$ \\
\hline & & Considerar importante la educación & $60 \%$ & $48 \%$ \\
\hline & & Participar en esos procesos educativos & $30 \%$ & $27 \%$ \\
\hline & & Respetar el medio ambiente & $61 \%$ & $45 \%$ \\
\hline & & Saber "mucho" o "algo" acerca del CC & $45 \%$ & $61 \%$ \\
\hline \multirow{7}{*}{$\begin{array}{l}\text { Rodríguez et al. } \\
\text { (2010) }\end{array}$} & \multirow{7}{*}{$\begin{array}{l}\text { Ideas/creencias sobre } \\
\text { acciones para la } \\
\text { mitigación del CC }\end{array}$} & Intención de tomar acciones & $* *$ & \\
\hline & & Reciclar* & ** & \\
\hline & & $\begin{array}{l}\text { Usar transporte público en vez de automóvil } \\
\text { privado }\end{array}$ & ** & \\
\hline & & $\begin{array}{l}\text { Pagar más por comida obtenida sin } \\
\text { fertilizantes }\end{array}$ & ** & \\
\hline & & Comer menos carne & ** & \\
\hline & & Votar por leyes ambientales & ** & \\
\hline & & Apoyar impuestos ambientales & ** & \\
\hline $\begin{array}{c}\text { Dijkstra y } \\
\text { Goedhart (2011) }\end{array}$ & $\begin{array}{l}\text { Disposición hacia la } \\
\text { ciencia }\end{array}$ & $\begin{array}{l}\text { Los estudios sobre CC son importantes para } \\
\text { el futuro }\end{array}$ & * & \\
\hline \multirow{6}{*}{$\begin{array}{l}\text { Kilınç et al. } \\
\quad(2011)\end{array}$} & \multirow{6}{*}{$\begin{array}{l}\text { Ideas/creencias sobre } \\
\text { acciones para la } \\
\text { mitigación del CC }\end{array}$} & Respetar el medio ambiente & $82 \%$ & $76 \%$ \\
\hline & & Pagar más por electricidad nuclear & $60 \%$ & $54 \%$ \\
\hline & & Comprar electrodomésticos eficientes & $76 \%$ & $80 \%$ \\
\hline & & Pagar para plantar árboles & $91 \%$ & $88 \%$ \\
\hline & & Reducir el consumo de carne en la dieta & $37 \%$ & $28 \%$ \\
\hline & & Saber "mucho" o "algo" acerca de CC & $82 \%$ & $76 \%$ \\
\hline
\end{tabular}




\begin{tabular}{|c|c|c|c|c|}
\hline \multirow{5}{*}{$\begin{array}{l}\text { Malandrakis et } \\
\text { al. (2011) }\end{array}$} & \multirow{5}{*}{$\begin{array}{l}\text { Ideas/creencias sobre } \\
\text { acciones para la } \\
\text { mitigación del CC }\end{array}$} & $\begin{array}{l}\text { Pagar más por comida obtenida sin } \\
\text { fertilizantes }\end{array}$ & $73 \%$ & $62 \%$ \\
\hline & & Reducir el consumo de carne en la dieta & $35 \%$ & $27 \%$ \\
\hline & & $\begin{array}{l}\text { Recibir formación en educación ambiental } \\
\text { adicional }\end{array}$ & $59 \%$ & $53 \%$ \\
\hline & & Usar automóviles más pequeños y eficientes & & ** \\
\hline & & Comprar electrodomésticos de bajo consumo & & ** \\
\hline \multirow{5}{*}{$\begin{array}{l}\text { ***Ambusaidi et } \\
\text { al. (2012) }\end{array}$} & \multirow{5}{*}{$\begin{array}{l}\text { Ideas/creencias sobre } \\
\text { acciones para la } \\
\text { mitigación del CC }\end{array}$} & Comprar electrodomésticos más eficientes & $71 \%$ & $78 \%$ \\
\hline & & Usar automóviles más pequeños & $45 \%$ & $52 \%$ \\
\hline & & Pagar para plantar árboles & $77 \%$ & $72 \%$ \\
\hline & & Participar en procesos educativos & $81 \%$ & $78 \%$ \\
\hline & & Saber "mucho" o "algo" del CC & $75 \%$ & $70 \%$ \\
\hline \multirow{6}{*}{$\begin{array}{c}\text { Boyes y } \\
\text { Stanisstreet } \\
(2012)\end{array}$} & \multirow{6}{*}{$\begin{array}{c}\text { Creencias sobre } \\
\text { acciones para la } \\
\text { mitigación del CC }\end{array}$} & Apagar los dispositivos electrónicos sin uso & $71 \%$ & $65 \%$ \\
\hline & & Reducir el uso del automóvil & $23 \%$ & $15 \%$ \\
\hline & & Reducir el consumo de carne & $27 \%$ & $15 \%$ \\
\hline & & Utilizar electrodomésticos de bajo consumo & $40 \%$ & $33 \%$ \\
\hline & & Comprar menos productos de moda nuevos & $31 \%$ & $26 \%$ \\
\hline & & $\begin{array}{l}\text { Tener en cuenta las políticas ambientales en } \\
\text { el voto }\end{array}$ & $48 \%$ & $38 \%$ \\
\hline \multirow{2}{*}{$\begin{array}{c}\text { Barros y } \\
\text { Pinheiro (2013) }\end{array}$} & \multirow{2}{*}{ Comprensión del CC } & Apoyar acciones individuales y colectivas & $* *$ & \\
\hline & & $\begin{array}{l}\text { Apoyar medidas de conservación del medio } \\
\text { ambiente }\end{array}$ & $48 \%$ & $35 \%$ \\
\hline \multirow{2}{*}{$\begin{array}{l}\text { Özdem et al. } \\
\quad(2014)\end{array}$} & \multirow{2}{*}{$\begin{array}{l}\text { Preocupaciones, } \\
\text { actitudes, acciones }\end{array}$} & Comprar bombillas más eficientes & $37 \%$ & $27 \%$ \\
\hline & & Reciclar* & $73 \%$ & $62 \%$ \\
\hline \multirow{7}{*}{$\begin{array}{c}\text { Hermans y } \\
\text { Korhonen (2017) }\end{array}$} & \multirow{7}{*}{$\begin{array}{l}\text { Actitudes, visiones y } \\
\text { disposición a actuar }\end{array}$} & Apagar los dispositivos electrónicos sin uso & $* *$ & \\
\hline & & Tomar duchas más cortas & ** & \\
\hline & & Apagar las luces de habitaciones sin uso & $* *$ & \\
\hline & & $\begin{array}{l}\text { Andar y desplazarse en bicicleta en distancias } \\
<5 \mathrm{~km}\end{array}$ & $* *$ & \\
\hline & & Bajar la temperatura dentro de casa & $* *$ & \\
\hline & & Comprar artículos de segunda mano & $* *$ & \\
\hline & & Comprar menos ropa y otras cosas & & ** \\
\hline
\end{tabular}




\begin{tabular}{|c|c|c|c|}
\hline \multirow{8}{*}{$\begin{array}{c}\text { Hermans y } \\
\text { Korhonen (2017) } \\
\text { (cont.) }\end{array}$} & \multirow{8}{*}{$\begin{array}{l}\text { Actitudes, visiones y } \\
\text { disposición a actuar } \\
\text { (cont.) }\end{array}$} & Reducir el consumo de carne & $* *$ \\
\hline & & Vender cosas de segunda mano & $* *$ \\
\hline & & Evitar el uso de vehículos a motor & ** \\
\hline & & $\begin{array}{l}\text { Puede ser mitigado mediante cooperación } \\
\text { internacional }\end{array}$ & $* *$ \\
\hline & & La mitigación es necesaria & ** \\
\hline & & $\begin{array}{l}\text { Todos y todas, como individuos debemos } \\
\text { actuar }\end{array}$ & $* *$ \\
\hline & & La mitigación es posible & $* *$ \\
\hline & & $\begin{array}{l}\text { La mitigación puede ser a través de acciones } \\
\text { políticas }\end{array}$ & $* *$ \\
\hline \multirow{2}{*}{$\begin{array}{l}\text { Stevenson et al. } \\
\qquad(2017)\end{array}$} & \multirow{2}{*}{$\begin{array}{l}\text { Creencias y } \\
\text { percepciones }\end{array}$} & Apoyar medidas de mitigación y adaptación & $* *$ \\
\hline & & Respetar el medio ambiente & ** \\
\hline
\end{tabular}

Nota: Se presentan los resultados que ofrecieron diferencias estadísticamente significativas para $\mathrm{p}<0,05$.

* El contenido expresa un conocimiento errado o concepción alternativa.

** El estudio no ofrece los porcentajes desagregados, pero indica que un mayor número de mujeres/hombres indicó esta opción.

*** En Omán las aulas son diferenciadas por sexo.

Fuente: Elaboración propia

\section{Percepciones y valoraciones personales}

La Tabla 4 presenta la información de investigaciones que mostraron diferencias estadísticamente significativas al indagar acerca de las percepciones y valoraciones personales en función del género.

Tabla 4

Estudios en los que se obtuvieron diferencias en percepciones y valoraciones en función del género

\begin{tabular}{clcc} 
Estudio & \multicolumn{1}{c}{ Percepciones } & M & $\mathrm{H}$ \\
\hline Chhokar et al. (2011) & Preocupación por el CC & $\mathbf{9 3 \%}$ & $88 \%$ \\
\hline Kilınç et al. (2011) & Preocupación por el CC & $\mathbf{9 5 \%}$ & $88 \%$ \\
\hline Malandrakis et al. (2011) & Confianza en los acuerdos internacionales & $\mathbf{6 4 \%}$ & $54 \%$ \\
\hline Barros y Pinheiro (2013) & Mayor emotividad & $* *$ & \\
\hline Özdem et al. (2014) & Preocupación por la extinción de especies* & $\mathbf{2 0 \%}$ & $13 \%$ \\
\cline { 2 - 4 } & Preocupación por los desechos radioactivos* & $16 \%$ & $\mathbf{2 4 \%}$ \\
\cline { 2 - 4 } & Preocupación por la basura* & $30 \%$ & $\mathbf{4 1 \%}$ \\
\hline Stevenson et al. (2014) & Mayor riesgo & $* *$ & \\
\hline Stevenson y Peterson (2016) & Mayor preocupación & $* *$ & \\
\cline { 2 - 4 } & Mayor esperanza & $* *$ \\
\hline Stevenson et al. (2016) & Mayor preocupación & \\
\hline
\end{tabular}




\begin{tabular}{cll}
\hline \multirow{2}{*}{ Hermans y Korhonen (2017) } & CC como un riesgo & $* *$ \\
\cline { 2 - 3 } & Confianza en la mitigación & $* *$ \\
\hline \multirow{2}{*}{ Stevenson et al. (2017) } & Mayor preocupación & $* *$ \\
\cline { 2 - 3 } & Mayor esperanza & $* *$ \\
\hline
\end{tabular}

Nota: Se presentan los resultados que ofrecieron diferencias estadísticamente significativas para $\mathrm{p}<0,05$.

* El contenido expresa un conocimiento errado o concepción alternativa.

** El estudio no ofrece los porcentajes desagregados, pero indica que un mayor número de mujeres/hombres indicó esta opción.

Fuente: Elaboración propia

\section{Procesos de enseñanza-aprendizaje}

En relación con este apartado, solo dos de los estudios exploraron cómo determinadas intervenciones educativas tienen efectos sobre la población estudiantil y las valoraciones de los estudiantes en función del género. En la revisión documental se indicaba que algunos hallazgos sugerían que las metodologías clásicas empleadas en la enseñanza de las ciencias no resultaban atractivas para las mujeres. Al respecto, Dijkstra y Goedhart (2011) sugieren que estas prácticas son menos interesantes para las mujeres y en su estudio comprueban cómo el trabajo por proyectos es valorado de forma más positiva por las adolescentes que por los muchachos, pese a que ellos evalúan la realización de este tipo de actividades colectivas como más fáciles. Por otro lado, Bodzin y Fu (2014) implementaron el denominado currículo geoespacial, en el que utilizaron herramientas tecnológicas y enfoques basados en la alfabetización científica o geoespacial. Los resultados reportaron que las mujeres presentaban una menor confianza y aprendizajes con este tipo de procesos de enseńanza.

Acceso a información relativa al CC. En el compendio de artículos analizados, pocos indagaron en la forma en que los adolescentes acceden a información de este tópico. Por ejemplo, Boyes et al. (2008) indican que el 41\% de los participantes declararon usar con frecuencia los medios de comunicación para informarse acerca del CC, siendo superior el porcentaje de hombres (46\%) que de mujeres (33\%); un mayor porcentaje de varones indicó hacerlo ocasionalmente (90\% de hombres frente a un $84 \%$ de mujeres). Por otro lado, Lin (2017) obtuvo en sus resultados que las mujeres preferían, en mayor medida, recibir información acerca del CC en los libros de texto.

Evolución en el transcurso escolar. A pesar de que muchos estudios contaron con población escolar de diferentes niveles educativos, los resultados presentados no aparecen desagregados por sexo y curso, circunstancia que dificulta generar una idea clara de cómo influye la cantidad de tiempo escolar en la brecha de género documentada por otras investigaciones. A pesar de ello, el transcurso escolar junto con el desarrollo evolutivo y otros procesos de socialización fuera del contexto escolar parecen influir en las percepciones y valoraciones relativas al CC y el medio ambiente. Al respecto, Boyes et al. (2009) obtuvieron diferencias estadísticamente significativas en las valoraciones de los estudiantes en cuanto al grado en que se consideraban respetuosos con el medio ambiente, disminuyendo de modo inversamente proporcional al nivel educativo (de los 12 a los 16 ańos); aunque como indican los autores, ello puede deberse a que los estudiantes de mayor edad son más conscientes de la crisis ambiental y, por tanto, sus juicios resultan más severos. Los autores se apoyan en que existen ítems como "el uso de automóviles pequeños" o "usar transporte público", que representan acciones más cercanas a los estudiantes de mayor edad, en las que sí se incrementa el porcentaje de respuesta positiva en función de la edad. 
Uno de los estudios que correlacionó las variables de género y el nivel educativo fue el de Rodríguez et al. (2010), que contó con participantes de entre 12 y 16 años. Los autores encontraron diferencias significativas entre las mujeres más jóvenes, quienes estaban dispuestas a comprar menos productos nuevos y evitar tirar basura en la playa; mientras que las de más edad indicaron — en mayor porcentaje — estar dispuestas a pagar más por una casa bien aislada. Igualmente en esta investigación las mujeres más jóvenes declararon —en mayor medida que los varones de su edad- que no tirar basura en la playa y pagar tasas ambientales son medidas efectivas frente al CC, mientras que un mayor número de hombres del mismo nivel educativo consideró que el aire acondicionado fue un paso positivo para el medio ambiente. Por otro lado, las adolescentes de mayor edad indicaron que utilizar electrodomésticos de bajo consumo constituía una acción eficaz frente al CC, mientras que sus homólogos masculinos manifestaron que votar acuerdos internacionales comporta una buena dirección para encaminar medidas de mitigación.

\section{Discusión}

Los resultados del metaanálisis del compendio de 26 artículos seleccionados de la RBS acerca de la comprensión del CC por parte de estudiantes de entre 12 y 18 ańos confirman algunas de las tendencias y hallazgos que sugiere la literatura sobre educación ambiental y las enseńanzas de las ciencias.

Este análisis solo identifica tres estudios — de los 26- donde no se encontraron diferencias significativas entre el género de los participantes. En dos de ellos la población objetivo incluía estudiantes de niveles educativos primarios (10-12 años) y secundarios (12-16), por lo que un rango de edad inferior puede influir en la reducción de las diferencias de género respecto de ciertos conocimientos y disposiciones hacia la ciencia, los que se refuerzan en función de diversos procesos de socialización dentro y fuera de la escuela durante la adolescencia.

El resto de los estudios sí ofrecen diferencias significativas que corroboran algunos de los hallazgos de otros estudios en el ámbito de la enseñanza de las ciencias y de la educación ambiental.

En el ámbito de los conocimientos, la literatura sugiere que las mujeres demuestran poseer menos conocimientos —o muestran menos interés - en cuestiones del ámbito científico-tecnológico (Fisher et al., 2020), aunque también evidencian más valores ecológicos (McCright, 2010).

En el caso de nuestro metaanálisis, en la categoría de conocimientos conceptuales (Tabla 2), los hombres obtienen mejores resultados — en el 24,39\% de los datos registrados_-, donde dos de ellos están relacionados con resultados de una prueba de conocimiento general de CC. Los otros ocho ítems se relacionan con contenidos específicos de acciones para la mitigación, por ejemplo: reducir el uso de petróleo y carbón; usar vehículos eléctricos y energías renovables; aumentar la generación de energía nuclear; comprar menos productos de moda nuevos, comer menos carne y plantar árboles. Sin embargo, las mujeres ofrecieron mejores resultados en 21 de las diferencias significativas reportadas $(51,21 \%)$ sobre diversos tópicos relacionados con el CC entre los que destacan: la importancia de la educación y del aumento de legislación ambiental e intervenciones de educación ambiental para luchar frente al CC; la reducción de consumo energético doméstico; la desconexión de dispositivos que no se usen; el uso de electrodomésticos más eficientes; la mejora del asilamiento del hogar o la reducción del uso de fertilizantes artificiales para producir alimentos; usar automóviles más pequeños y eficientes, y aumentar el sistema de producción nuclear. De este modo, en los contenidos relacionados con la energía nuclear, los hombres puntúan con mayor acierto (Ambusaidi et al., 2012), mientras que en otros contextos destacan las mujeres (Boyes et al., 2009; Daniel et al., 2004; Kılınç et al., 2011). 
En el ámbito científico-tecnológico estricto, las mujeres consiguieron mejores resultados en tópicos relacionados con el aumento de temperatura del planeta, la intensificación de fenómenos meteorológicos extremos o la afectación sobre la producción agrícola. Pese a ello, también fueron las mujeres las que declararon poseer un mayor número de concepciones alternativas ${ }^{3}$ (Cubero, 1994) o teorías implícitas (Rodríguez y González, 1995) como reducir la contaminación marina, la basura de los ríos o las emisiones de CFCs constituyen medidas efectivas frente al $\mathrm{CC}$, al igual que proteger especies en peligro de extinción, reciclar o usar gasolina sin plomo.

En referencia a los conocimientos actitudinales y las diferencias estadísticamente significativas reportadas en los estudios (Tabla 3), los hombres solo indican una mayor disposición a actuar que las mujeres en ocho de 58 diferencias significativas encontradas y se relacionan con comprar electrodomésticos eficientes y menos ropa, así como usar automóviles más pequeños y eficientes.

Por su parte, las mujeres están más dispuestas a pagar más por una casa bien aislada, alimentos producidos sin fertilizantes artificiales, energía nuclear o plantar árboles; comprar y vender artículos de segunda mano y comprar menos artículos de moda; utilizar electrodomésticos de bajo consumo; bajar la temperatura dentro de casa; usar transporte público; andar o usar la bicicleta en lugar del automóvil privado; modificar la dieta para consumir menos carne; reciclar; y apagar los dispositivos que no se utilicen o tomar duchas más cortas. Igualmente, ellas otorgan mayor importancia y relevancia a la educación en general y a la educación ambiental en particular, así como a la participación en los procesos educativos; el respeto al medio ambiente a través de la política y la legislación ambiental, y la mitigación y adaptación. También muestran mayor confianza en las acciones individuales y colectivas, así como en la cooperación internacional.

En el ámbito de las percepciones y las valoraciones personales (Tabla 4) los resultados muestran la tendencia de la literatura científica. Así, las mujeres declaran: mayor grado de preocupación que los hombres (Chhokar et al., 2011; Kılınç et al., 2011; Stevenson \& Peterson, 2016; Stevenson et al., 2016; Stevenson et al., 2017); mayores niveles de esperanza (Stevenson \& Peterson, 2016; Stevenson et al., 2017); mayor grado de percepción de riesgo (Hermans \& Korhonen, 2017; Stevenson et al., 2014); y mayor grado de emotividad (Barros \& Pinheiro, 2013). Los hombres solo muestran diferencias en su favor en aspectos relacionados con la preocupación por los residuos radiactivos y basuras; dos concepciones alternativas por su falta de conexión directa con las causas o las consecuencias del CC y que se obtuvieron en el estudio de Özdem et al. (2014) con estudiantes de 12-13 años, correspondiente al nivel inferior de la educación secundaria donde los patrones y comportamientos diferenciados por el género no están todavía tan marcados. Por otro lado, el estudio de Hermans y Korhonen (2017) identifica que los hombres muestran una mayor confianza en la mitigación, lo que podría explicarse por la confianza demostrada en relación con la ciencia y la tecnología como solución a la crisis climática (Jenkins \& Pell, 2006; Schönfelder \& Bogner, 2020).

Así, los resultados corroboran las conclusiones de la literatura revisada, donde las mujeres muestran diferencias significativas en elementos que se enmarcarían en cosmovisiones más ecocéntricas y comunitaristas que los hombres; otorgando respuestas más coherentes con una representación adecuada de la crisis climática (Cantell, Tolppanen, Aarnio-Linnanvuori \& Lehtonen, 2019). Por el contrario, existen contenidos específicos - como los relacionados con la energía nuclear, elemento que podríamos situar en una cosmovisión tecnicista- que, dependiendo del estudio, muestra unos resultados u otros en función del género, siendo necesario explorarlos con mayor profundidad para comprender la razón de esta ambivalencia.

3. Utilizamos la terminología "concepción alternativa” para denotar una idea, concepción, representación o conocimiento que no guarda coherencia con el conocimiento científico. El calificativo "alternativa” no pretende establecer un estatus entre el conocimiento científico y el conocimiento común, ni connotar calificativos como ingenuo o erróneo. 
En relación con las diferencias encontradas, y su reproducción y agravamiento a lo largo del transcurso de la educación secundaria, pareciera que las diferencias de género aparecen y se refuerzan en este nivel educativo/ evolutivo. Por un lado, y de forma esperada, la adquisición y posesión de conocimientos parecen incrementarse proporcionalmente con el avance de los cursos (Boyes et al., 2008); sin embargo, las diferencias de género también aumentan — como sugieren los estudios de Harker-Schuch y Bugge-Henriksen (2013) y Yazdanparast et al. (2013) — con el alumnado de entre 16 y 17 años y de entre 15 y 17 años respectivamente, donde los hombres obtuvieron una mayor puntuación total en un cuestionario referente a conocimientos generales del CC. Sucede lo mismo - pero de forma inversa - en el ámbito de las percepciones, donde las diferencias de género en relación con los niveles de preocupación, responsabilidad y disposición a actuar de manera ambientalmente respetuosa disminuyen proporcionalmente con la edad. En esta línea, Chokkar et al. (2011) obtuvieron una fuerte relación entre los niveles educativos superiores y el respeto hacia el medio ambiente, con una disminución del porcentaje de estudiantes de niveles superiores que indicaron sentirse "muy" o "bastante" respetuosos con el medio. Una tendencia similar a la registrada por Boyes et al. (2009) entre los 12 y los 16 años, aunque — como indicábamospodría ser debido a una mayor exigencia de juicio entre los estudiantes de mayor edad (Boyes et al., 2009).

Por otro lado, en el estudio de Stevenson et al. (2016) en el que también participaron 110 estudiantes de niveles primarios de entre 11 y 14 años — correspondiente al 25\% de la muestra- sugieren que la tendencia de una mayor preocupación por el CC por parte de las mujeres se mantiene tanto entre jóvenes como en adultos. Estos resultados comparados con los estudios consultados sugieren que los procesos de socialización de género acompañan —e interaccionan - con los procesos socioeducativos, esto es, en la disposición hacia ciertos contenidos científico-técnicos, en relación con una mayor o menor disposición hacia comportamientos proambientales/ climáticos y en el ámbito de las percepciones, ya sean de riesgo, de preocupación, responsabilidad o confianza.

\section{Conclusión}

Con este estudio pretendíamos explorar y sintetizar los resultados ofrecidos por la literatura científica respecto de las posibles diferencias de género existentes entre estudiantes adolescentes (12-18 años) en cuanto al conocimiento y representación del CC. Para ello, se han identificado y sintetizado los hallazgos aportados por la literatura científica en este campo de estudio. Así, los resultados refuerzan las conclusiones del proyecto de investigación del que forma parte este estudio (Meira-Cartea, Bisquert, García-Vinuesa y Pérez, 2018), que pretende comprender cómo representan el CC los y las estudiantes de educación secundaria.

En este sentido, y considerando que el objetivo de este estudio no trata de valorar los logros educativos de los diversos sistemas educativos, los resultados actuales sugieren que los enfoques educativos para enfrentar el reto climático — ya sean desde las diferentes versiones que encajan en el amplio espectro entre el paradigma positivista y el constructivista (Busch, Henderson \& Stevenson, 2019) — parecen haber alcanzado un techo como respuesta educativa al CC.

En este sentido, la crisis climática se configura como un contenido estanco del campo científico de las ciencias y la tecnología, sin establecer la necesaria vinculación con sus causas socioeconómicas y culturales, así como con los riesgos que entrańan para la población mundial los desastres humanitarios y el agravamiento de la vulnerabilidad de determinados grupos sociales. Esta visión refuerza concepciones alternativas en relación con la explicación del fenómeno y que — como parece indicar este análisis - reproduce patrones discriminatorios de género. Una circunstancia que limita las habilidades esenciales sobre las que debemos educarnos para afrontar una agenda de descarbonización — ya retrasada - (González-Gaudiano y Meira-Cartea, 2020) que promueva 
una ecociudadanía que participe, exija y acepte las urgentes medidas y políticas que la comunidad científica lleva reclamando desde mediados del siglo pasado, esto es, acciones orientadas a reducir drásticamente la emisión de gases de efecto invernadero y nuestro impacto en la biosfera.

Difícilmente estos cambios necesarios se pueden lograr desde una visión científica positivista de la educación para el cambio climático ya que, aunque la ciencia explica sus causas en las interacciones de elementos físico-químicos, estas radican en estilos de vida hiperconsumistas y dependientes de combustibles fósiles de las sociedades más industrializadas. Para ello, sería preciso una reforma transformadora que facilite la transición ecológica en todos los sectores económicos más afectados (energía, automoción, transporte aéreo, alimentación, etc.) acompańada por una innovación educativa hacia una paradigma sociocrítico y transformador que permita acompasar los necesarios cambios económicos y culturales que exige la crisis climática (Busch et al., 2019).

Así, parece necesario reflexionar acerca de la idoneidad de determinadas prácticas educativas que fomentan el desinterés y la desafección hacia los conocimientos científicos y ambientales de la mitad de la población estudiantil (las mujeres); pero también la persistencia en los estudiantes varones de actitudes y percepciones erróneas para mitigar y adaptarnos al CC. Dos ámbitos de la representación en los que encontramos diferencias de género que deben ser erradicadas, ya que mantener enfoques alfabetizadores como respuesta socioeducativa para la crisis climática comporta situaciones de discriminación, reforzadas por otros procesos socializadores fuera de la escuela, donde la mitad de la población estudiantil (los varones) parece saber e interesarse más en la ciencia. Sin embargo, aunque las mujeres presentan menores logros respecto de la adquisición de conocimiento científico, representan más coherentemente la crisis climática en lo referido a percepción de riesgo, responsabilidad en sus causas y respuestas acordes con valores ambientales y comunitarios.

Estos resultados sugieren que la posesión y aplicación de conocimientos científicos, junto con los procesos de socialización de género, no facilitan una percepción del problema coherente con el conocimiento científico y de ahí que resulte ineficaz desde el punto de vista de la adaptación y de la resiliencia.

Al igual que en cualquier ámbito de la ciencia es necesario desarrollar un espíritu crítico que cuestione las verdades absolutas, y que contraste y elabore — a través del método científico- hipótesis que permitan resolver problemas y avanzar en el desarrollo humano; de ahí la necesidad de una sociedad que cuestione y contraste tradiciones, roles y estereotipos de género reproducidos por un sistema históricamente patriarcal (Lagarde, 1997). Desde esta perspectiva, "feminizar" los espacios de socialización primaria, así como los espacios educativos no debe significar un ataque hacia los hombres, sino una propuesta que permita contrastar los patrones y estereotipos masculinos para avanzar en la reconstrucción de nuevas identidades, en las que el respeto y los cuidados hacia el medio ambiente y el resto de seres vivos no sean conductas exclusivas de las mujeres y poco atractivas para los hombres.

Además, se comprueba que los enfoques situados en la teoría del déficit de conocimiento y que justifican su utilidad basándose en la premisa de que un mejor conocimiento produce una conducta más adecuada y coherente no constituyen una respuesta socioeducativa adecuada para la crisis ambiental (González-Gaudiano \& Meira-Cartea, 2019). Los resultados no apoyan esta premisa y sugieren que conforme los estudiantes avanzan de nivel educativo, y bajo el supuesto de que han adquirido conocimientos más adecuados, complejos y avanzados, estos no influyen en comportamientos como la autoeficacia, la motivación o las percepciones de responsabilidad y riesgo. Este análisis revela que la edad influye en las actitudes positivas hacia el medio ambiente, una situación desesperanzadora, pero que dibuja un camino para la atención profesional de educadoras, educadores y ecociudadanas. 
En definitiva, ante una situación de riesgo que ya es intemporal, existen registros y experiencias — pasadas y presentes- y previsiones de futuro que confirman el agravamiento de tales escenarios. Ante esto y un CC machista, racista y clasista producido por un sistema socioeconómico que prioriza los beneficios económicos por sobre los desastres humanos, urge promover una transformación estructural y cultural, cimentada en el ecofeminismo y en la justicia climática y social.

El artículo original fue recibido el 14 de mayo de 2020 El artículo revisado fue recibido el 22 de julio de 2020

El artículo fue aceptado el 3 de agosto de 2020

\section{Referencias}

Ambusaidi, A., Boyes, E., Stanisstreet, M., \& Taylor, N. (2012). Omani students' views about global warming: Beliefs about actions and willingness to act. International Research in Geographical and Environmental Education, 21(1), 21-39. https://doi.org/10.1080/10382046.2012.639154

Barros, H. C. L. \& Pinheiro, J. Q. (2013). Dimensões psicológicas do aquecimento global conforme a visão de adolescentes brasileiros. Estudos de Psicologia (Natal), 18(2), 173-182. https://doi.org/10.1590/s1413-294x2013000200002

Bodzin, A. M. \& Fu, Q. (2014). The effectiveness of the geospatial curriculum approach on urban middle-level students' climate change understandings. Journal of Science Education and Technology, 23(4), 575-590. https://doi.org/10.1007/s10956-013-9478-0

Boyes, E., Chuckran, D., \& Stanisstreet, M. (1993). How do high school students perceive global climatic change: What are its manifestations? What are its origins? What corrective action can be taken? Journal of Science Education and Technology, 2(4), 541-557. https://doi.org/10.1007/bf00695323

Boyes, E., Skamp, K., \& Stanisstreet, M. (2009). Australian secondary students' views about global warming: Beliefs about actions, and willingness to act. Research in Science Education, 39(5), 661-680. https://doi.org/10.1007/s11165-008-9098-5

Boyes, E. \& Stanisstreet, M. (2012). Environmental education for behaviour change: Which actions should be targeted? International Journal of Science Education, 34(10), 1591-1614. https://doi.org/10.1080/09500693.2011.584079

Boyes, E., Stanisstreet, M., \& Yongling, Z. (2008). Combating global warming: The ideas of high school students in the growing economy of South East China. International Journal of Environmental Studies, 65(2), 233-245. https://doi.org/10.1080/00207230701284543

Bozdoğan, A. E. (2011). A collection of studies conducted in education about "global warming" problem. Educational Sciences: Theory \& Practice, 11(3), 1618-1624. Recuperado de https://files.eric.ed.gov/fulltext/EJ936338.pdf

Busch, K. C., Henderson, J. A., \& Stevenson, K. T. (2019). Broadening epistemologies and methodologies in climate change education research. Environmental Education Research, 25(6), 955-971. https://doi.org/10.1080/13504622.2018.1514588

Cantell, H., Tolppanen, S., Aarnio-Linnanvuori, E., \& Lehtonen, A. (2019). Bicycle model on climate change education: Presenting and evaluating a model. Environmental Education Research, 25(5), 717-731. https://doi. org/10.1080/13504622.2019.1570487

Cheryan, S., Plaut, V. C., Davies, P. G., \& Steele, C. M. (2009). Ambient belonging: How stereotypical cues impact gender participation in computer science. Journal of Personality and Social Psychology, 97(6), 1045. https://doi. org/10.1037/a0016239

Chhokar, K., Dua, S., Taylor, N., Boyes, E., \& Stanisstreet, M. (2011). Indian secondary students' views about global warming: Beliefs about the usefulness of actions and willingness to act. International Journal of Science and Mathematics Education, 9(5), 1167-1188. https://doi.org/10.1007/s10763-010-9254-z

Creswell, J. (2013). Qualitative inquiry and research design: Five different approaches. California: SAGE. 
Cubero, R. (1994). Concepciones alternativas, preconceptos, errores conceptuales... ¿distinta terminología y un mismo significado? Investigación en la Escuela, 23, 33-42. Recuperado de https://idus.us.es/bitstream/handle/11441/59603/R23_3.pdf?sequence=1

Daniel, B., Stanisstreet, M., \& Boyes, E. (2004). How can we best reduce global warming? School students' ideas and misconceptions. International Journal of Environmental Studies, 61(2), 211-222. https://doi.org/10.1080/0020723032000087907

Dijkstra, E. M. \& Goedhart, M. J. (2011). Evaluation of authentic science projects on climate change in secondary schools: A focus on gender differences. Research in Science \& Technological Education, 29(2), 131-146. https://doi.org/10.1080/02635143.2011.581631

Dijkstra, E. M. \& Goedhart, M. J. (2012). Development and validation of the ACSI: Measuring students's science attitudes, pro-environmental behaviour, climate change attitudes and knowledge. Environmental Education Research, 18(6), 733-749. https://doi.org/10.1080/13504622.2012.662213

Finucane, M. L., Slovic, P., Mertz, C. K., Flynn, J., \& Satterfield, T. A. (2000). Gender, race, and perceived risk: The "white male" effect. Health, Risk \& Society, 2(2), 159-172. https://doi.org/10.1080/713670162

Fisher, C. R., Thompson, C. D., \& Brookes, R. H. (2020). Gender differences in the Australian undergraduate STEM student experience: a systematic review. Higher Education Research \& Development, 1-14. https://doi.org/10.1080/07294360.2020.1721441

García-Vinuesa, A. y Meira-Cartea, P. Á. (2019). Caracterización de la investigación educativa sobre el cambio climático y los estudiantes de educación secundaria. Revista Mexicana de Investigación Educativa, 24 (81), 507-535. Recuperado de http://www.scielo.org.mx/pdf/rmie/v24n81/1405-6666-rmie-24-81-507.pdf

González-Gaudiano, E. J. \& Meira-Cartea, P. Á. (2019). Environmental education under siege: Climate radicality. The Journal of Environmental Education, 50(4-6), 386-402. https://doi.org/10.1080/00958964.2019.1687406

González-Gaudiano, E. J. y Meira-Cartea, P. Á. (2020). Educación para el cambio climático: ¿educar sobre el clima o para el cambio? Perfiles Educativos, 42(168), 157-174. https://doi.org/10.22201/iisue.24486167e.2020.168.59464

Harker-Schuch, I. \& Bugge-Henriksen, C. (2013). Opinions and knowledge about climate change science in high school students. Ambio, 42(6), 755-766. https://doi.org/10.1007/s13280-013-0388-4

Hermans, M. \& Korhonen, J. (2017). Ninth graders and climate change: Attitudes towards consequences, views on mitigation, and predictors of willingness to act. International Research in Geographical and Environmental Education, 26(3), 223-239. https://doi.org/10.1080/10382046.2017.1330035

Kılınç, A., Boyes, E., \& Stanisstreet, M. (2011). Turkish school students and global warming: Beliefs and willingness to act. Eurasia Journal of Mathematics, Science \& Technology Education, 7(2), 121-134. https://doi.org/10.12973/ejmste/75187

Jenkins, E. W. \& Pell, R. G. (2006). "Me and the environmental challenges": A survey of English secondary school students' attitudes towards the environment. International Journal of Science Education, 28(7), 765-780. https://doi.org/10.1080/09500690500498336

Lagarde, M. (1997). Género y feminismo: desarrollo humano y democracia. Madrid: Horas y Horas.

Liarakou, G., Athanasiadis, I., \& Gavrilakis, C. (2011). What Greek secondary school students believe about climate change? International Journal of Environmental and Science Education, 6(1), 79-98. Recuperado de https://www.pegem.net/dosyalar/dokuman/138390-20140102114018-5.pdf

Lin, J. (2017). Chinese grade eight students' understanding about the concept of global warming. EURASIA Journal of Mathematics, Science and Technology Education, 13(5), 1313-1330. https://oi.org/10.12973/eurasia.2017.00672a

Littell, J. H., Corcoran, J., \& Pillai, V. (2008). Systematic reviews and meta-analysis. Oxford: Oxford University Press.

Malandrakis, G., Boyes, E., \& Stanisstreet, M. (2011). Global warming: Greek students' belief in the usefulness of proenvironmental actions and their intention to take action. International Journal of Environmental Studies, 68(6), 947-963. https://doi.org/10.1080/00207233.2011.590720

McCright, A. M. (2010). The effects of gender on climate change knowledge and concern in the American public. Population and Environment, 32(1), 66-87. https://doi.org/10.1007/s11111-010-0113-1 
Meira-Cartea, P. Á., Bisquert, K. M., García-Vinuesa, A., y Pérez, A. (2018). RESCLIMA-EDU: A alfabetización climática en educación secundaria. Análise transcultural das representacións sociais do cambio climático en estudantes, docentes e material curricular. En A. Alonso-Ferreiro y A. Gewerc (Eds.), Conectando redes. La relación entre la investigación y la práctica educativa. Simposio REUNI+D y RILME (pp. 795-808). Santiago de Compostela: Grupo Stellae.

Monroe, M. C., Plate, R. R., Oxarart, A., Bowers, A., \& Chaves, W. A. (2019). Identifying effective climate change education strategies: A systematic review of the research. Environmental Education Research, 25(6), 791-812. https://doi.org/10.1080/13504622.2017.1360842

Oliva, J. M. (1999). Algunas reflexiones sobre las concepciones alternativas y el cambio conceptual. Enseñanza de las Ciencias: Revista de Investigación y Experiencias Didácticas, 17(1), 93-107. Recuperado de https://www.raco.cat/index.php/Ensenanza/article/view/21563

Özdem, Y., Dal, B., Öztürk, N., Sönmez, D., \& Alper, U. (2014). What is that thing called climate change? An investigation into the understanding of climate change by seventh-grade students. International Research in Geographical and Environmental Education, 23(4), 294-313. https://doi.org/10.1080/10382046.2014.946323

Petticrew, M. \& Roberts, H. (2008). Systematic reviews in the social sciences: A practical guide. Oxford: John Wiley \& Sons.

Rodríguez, A. y González, R. (1995). Cinco hipótesis sobre las teorías implícitas. Revista de Psicología General y Aplicada: Revista de la Federación Española de Asociaciones de Psicología, 48(3), 221-229. Recuperado de https://dialnet.unirioja.es/descarga/articulo/2161352.pdf

Rodríguez, M., Boyes, E., \& Stanisstreet, M. (2010). Spanish secondary students' willingness to undertake specific actions to combat global warming: Can environmental education help? Psyecology, 1(1), 73-89. https://doi.org/10.1174/217119710790709496

Rye, J. A., Rubba, P. A., \& Wiesenmayer, R. L. (1997). An investigation of middle school students' alternative conceptions of global warming. International Journal of Science Education, 19(5), 527-551. https://doi.org/10.1080/0950069970190503

Schönfelder, M. L. \& Bogner, F. X. (2020). Between science education and environmental education: How science motivation relates to environmental values. Sustainability, 12(5), 1968. https://doi.org/10.3390/su12051968

Serantes-Pazos, A. (2017). El cambio climático en los libros de texto: cómo el sistema educativo contribuye a la construcción del saber sobre el cambio climático. En M. Blanco \& P. Meira (Eds.), RESCLIMA: Aproximação ás claves sociais e educativas do cambio climático (pp. 81-91). Ferrol: Aldine Editorial.

Stevenson, K. T., King, T. L., Selm, K. R., Peterson, M. N., \& Monroe, M. C. (2017). Framing climate change communication to prompt individual and collective action among adolescents from agricultural communities. Environmental Education Research, 24(3), 365-377. https://doi.org/10.1080/13504622.2017.1318114

Stevenson, K. \& Peterson, N. (2016). Motivating action through fostering climate change hope and concern and avoiding despair among adolescents. Sustainability, 8(1), 1-10. https://doi.org/10.3390/su8010006

Stevenson, K. T., Peterson, M. N., \& Bondell, H. D. (2016). The influence of personal beliefs, friends, and family in building climate change concern among adolescents. Environmental Education Research, 25(6), 832-845. https://doi.org/10.1080/13504622.2016.1177712

Stevenson, K. T., Peterson, M. N., Bondell, H. D., Moore, S. E., \& Carrier, S. J. (2014). Overcoming skepticism with education: Interacting influences of worldview and climate change knowledge on perceived climate change risk among adolescents. Climatic change, 126(3-4), 293-304. https://doi.org/10.1007/s10584-014-1228-7

Stevenson, K. T., Peterson, M. N., \& Bradshaw, A. (2016). How climate change beliefs among US teachers do and do not translate to students. PLoS ONE, 11(9), 1-11, e0161462. https://oi.org/10.1371/journal.pone.0161462

Timulak, L. (2009). Meta-analysis of qualitative studies: A tool for reviewing qualitative research findings in psychotherapy. Psychotherapy Research, 19(4-5), 591-600. https://doi.org/10.1080/10503300802477989

Yazdanparast, T., Salehpour, S., Masjedi, M. R., Seyedmehdi, S. M., Boyes, E., Stanisstreet, M., \& Attarchi, M. (2013). Global warming: Knowledge and views of Iranian students. Acta Medica Iranica, 178-184. Recuperado de https://pubmed.ncbi.nlm.nih.gov/23605603/ 